\title{
The reproducibility of SfM algorithms to produce detailed Digital Surface Models: the example of PhotoScan applied to a high-alpine rock glacier
}

Hanne Hendrickx, Sebastián Vivero, Laure De Cock, Bart De Wit, Philippe De Maeyer, Christophe Lambiel, Reynald Delaloye, Jan Nyssen \& Amaury Frankl

To cite this article: Hanne Hendrickx, Sebastián Vivero, Laure De Cock, Bart De Wit, Philippe De Maeyer, Christophe Lambiel, Reynald Delaloye, Jan Nyssen \& Amaury Frankl (2019) The reproducibility of SfM algorithms to produce detailed Digital Surface Models: the example of PhotoScan applied to a high-alpine rock glacier, Remote Sensing Letters, 10:1, 11-20, DOI: 10.1080/2150704X.2018.1519641

To link to this article: https://doi.org/10.1080/2150704X.2018.1519641

\section{冊 Published online: 24 Oct 2018.}

\section{Submit your article to this journal $\pi$}

View Crossmark data ־ 


\title{
The reproducibility of SfM algorithms to produce detailed Digital Surface Models: the example of PhotoScan applied to a high-alpine rock glacier
}

\author{
Hanne Hendrickx ${ }^{a}$, Sebastián Vivero ${ }^{b}{ }^{b}$, Laure De Cock ${ }^{a}$, Bart De Wit ${ }^{a}$, \\ Philippe De Maeyer $\mathbb{D D}^{a}$, Christophe Lambiel ${ }^{b}$, Reynald Delaloye ${ }^{c}$, Jan Nyssen ${ }^{a}$ \\ and Amaury Frankl ${ }^{\mathrm{a}, \mathrm{d}}$
}

aDepartment of Geography, Ghent University, Ghent, Belgium; 'Institute of Earth Surface Dynamics, University of Lausanne, Lausanne, Switzerland; 'Department of Geosciences, University of Fribourg,

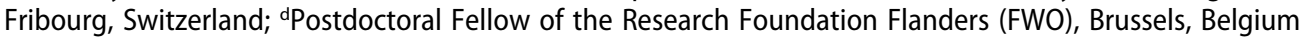

\begin{abstract}
In geomorphology, PhotoScan is a software that is used to produce Digital Surface Models (DSMs). It constructs 3D environments from 2D imagery (often taken by Unmanned Aerial Vehicles (UAV)) based on Structure-from-Motion (SfM) and Multi-View Stereo (MVS) principles. However, unpublished computer-vision algorithms used, contain random elements which can affect the accuracy of the outputs. For this letter, ten model runs with identical inputs were performed on UAV imagery of a rock glacier to analyse the magnitude of the variation between the different model outputs. This variation was quantified calculating the standard deviation of each cell value in the respective DSMs and derivatives (curvature). Places with steep slope gradients have considerably more DSM variation (up to $10 \mathrm{~cm}$ ) but stay within the range of the model's accuracy $(10$ vertical $\mathrm{cm}$ ) for 88 $96 \%$ of the area. The edges of the model also show a larger variability $(0.10-3 \mathrm{~m})$, related to a lower number of overlapping images. These results should be accounted for when performing a geomorphological research at centimetre scale using PhotoScan, especially in areas with a complex relief. Using medium-quality runs, additional oblique viewpoints and respecting a minimum of five overlapping images can minimize the software's variations.
\end{abstract}

\section{ARTICLE HISTORY}

Received 1 March 2018

Accepted 31 August 2018

\section{Introduction}

Structure-from-Motion (SFM) is a recent survey technique that merges novel advances in computer vision with digital photogrammetry procedures. This technique is widely used to construct 3D environments from 2D consumer grade images using the Multi-View Stereo (MVS) principle (Fonstad et al. 2013; Smith, Carrivick, and Quincey 2015). It has proven to be a successful technique in order to study forms and processes in geosciences (e.g., Niethammer et al. 2012; Javernick, Brasington, and Caruso 2014; Frankl et al. 2015; Piermattei et al. 2016; Dall'Asta et al. 2017) and yields accuracies similar to those of terrestrial laser scanning (Fonstad et al. 2013; Smith, Carrivick, and Quincey 2015). At landform and hillslope scales, Digital Surface 
Models (DSM) allow to investigate landforms at sub-centimetre accuracies (Harwin and Lucieer 2012; James, Robson, and Smith 2017), while processes (e.g., erosion and deposition) can be quantified at centimetre to sub-decimetre accuracies (e.g., Niethammer et al. 2012; Lannoeye et al. 2016; Piermattei et al. 2016; Dall'Asta et al. 2017). These accuracies highly depend on image acquisition distance (flight height), accuracy of the Ground Control Points (GCPs), camera properties (MegaPixels (MP), aperture, shutter speed, ISO and lens properties) and the texture contrast of the surface. Both ground-based and aerial images can be used. Since the recent innovation of Unmanned Aerial Vehicles (UAV), aerial imagery is becoming more frequently used, providing a low-cost and effective way to conduct high-resolution topographic surveys (Westoby et al. 2012).

PhotoScan Professional is a commercial software developed by Agisoft (Agisoft 2018) and is widely used in applying the SfM-MVS technique (e.g., Frankl et al. 2015; Uysal, Toprak, and Polat 2015; Lannoeye et al. 2016; Kenner et al. 2017). This software package identifies correspondences between features (key points) across overlapping images (Semyonov 2011), based on the Scale Invariant Feature Transform algorithm (SIFT, Lowe 2004). Estimated camera model parameters are used for self-calibration and to optimize camera position that then are refined after entering the GCPs and their coordinates, using a bundleadjustment algorithm. A dense point cloud, followed by a dense surface is then constructed. Finally, texture mapping is conducted (Semyonov 2011). However, extensive technical details about the algorithms used in Photoscan are unpublished.

To the best of our knowledge, the reproducibility of PhotoScan is not yet explicitly addressed by earlier technical papers. However, the software processing algorithms directly influence model accuracy (Uysal, Toprak, and Polat 2015; James et al. 2017a). Therefore, we explored the reproducibility of a 3D model of a complex alpine environment from multiple PhotoScan runs. This is done with exactly the same images, processing settings and GCPs as these input parameters have been identified influencing accuracies by previous research (Harwin and Lucieer 2012; James and Robson 2014; Uysal, Toprak, and Polat 2015; James, Robson, and Smith 2017). Results are discussed here and recommendations to future users are made.

\section{Study area, survey method and data processing}

\subsection{Study area}

The data was collected in October 2017 on the lower part of the Cliosses rock glacier, primarily composed of different sizes of angular metamorphic rocks. The study site is located on the right side of the Herens valley, Western Swiss Alps, at an elevation of 2400 - $2500 \mathrm{~m}$ a.s.l. (Figure 1). Surface velocities have been measured since 2006 and are lower than $0.5 \mathrm{~m}$ per year (Delaloye, Lambiel, and Gärtner-Roer 2010).

\subsection{UAV and camera specifications}

The UAV survey was performed in 2017 using a 16MP Panasonic Lumix DMC-GM5 equipped with a 12-32 mm F3.5-5.6 lens. The focal length was fixed to $24 \mathrm{~mm}$, a shutter speed of 1/500 and ISO set on 400. The UAV was a custom-made Hexacopter DJ F550 with a Pixhawk flight controller. In the first step of the flight planning, flight lines 


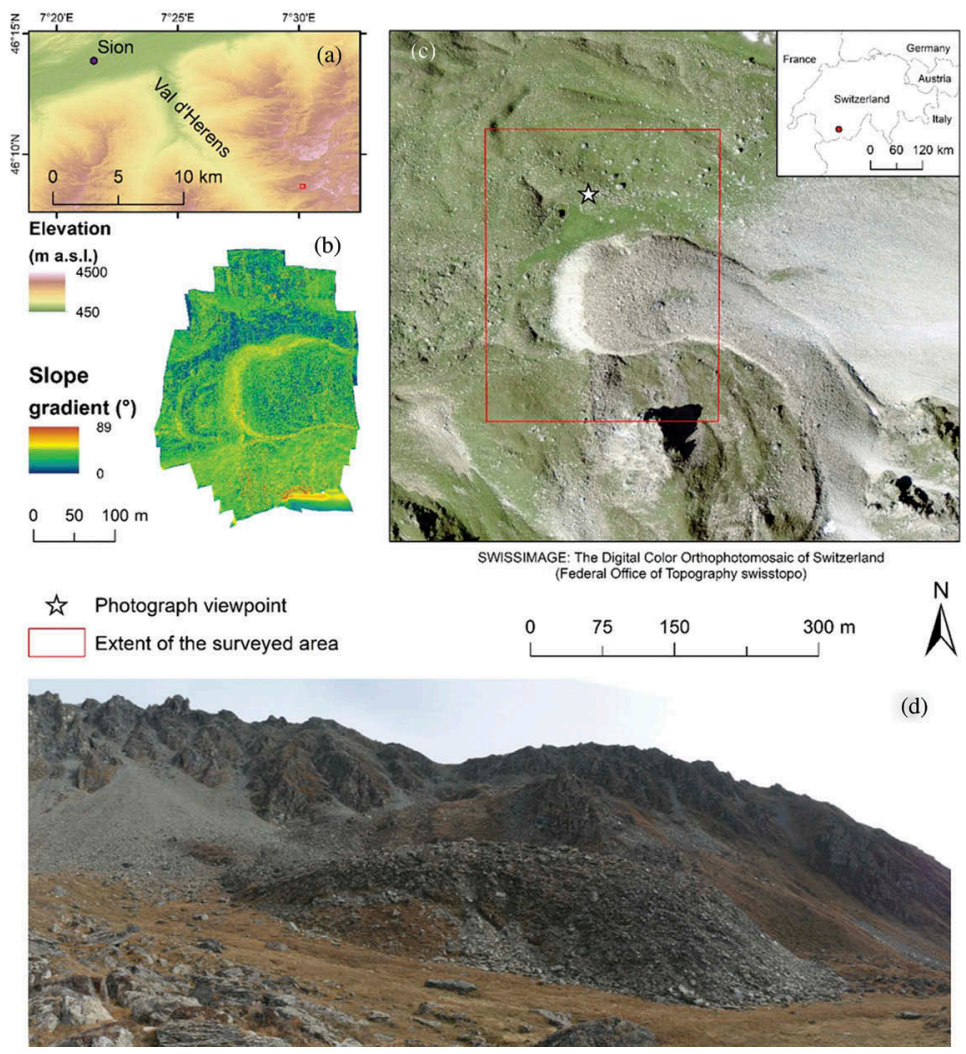

Figure 1. Situation of the study area (a): Les Cliosses rock glacier (c), including slope gradient (b) and a field photograph (d). Overall height of the rock glacier front is $25 \mathrm{~m}$.

were drawn in a GIS environment (QGIS 2.18.2), parallel to the contour lines of the area. A Python script was used to translate these flight lines into a waypoints-file. For every line, the script adjusted the flight height of the UAV according to the topography. This resulted in a constant flight height of approximately $90 \mathrm{~m}$ for every image. The speed of the UAV was 4-6 $\mathrm{m} \mathrm{s}^{-1}$ and the acquisition interval was set to $1 \mathrm{~s}$ to ensure abundant image overlap. This resulted in 561 images. To make this experiment quickly reproducible and avoiding long processing time, 24 images were used from five sequential flight lines, with a longitudinal overlap of $70 \%$ and a side overlap of $65-75 \%$. This resulted in an acquisition interval of $6 \mathrm{~s}$ (Figure 2). The ground pixel size and thus the Ground Sample Density (GSD) was $1.41 \mathrm{~cm} /$ pixel and an area of $64100 \mathrm{~m}^{2}$ was covered.

\section{3. dGNSS measurements}

Differential GNSS (Global Navigation Satellite System) measurements using Post-Processing Kinematics (PPK) were performed to reference the model to a real-world system. Within the small sample from the UAV survey used for this experiment, 8 ground control points (GCPs) and 13 check points (CPs, subset of existing dGNSS points measured for monitoring the rock glacier velocities) were used (Figure 2). The GCPs consisted of physical square targets $(0.5 \times 0.5 \mathrm{~m})$ with a high contrast and a clear centre (Figure 3(a)). The CPs are painted red 


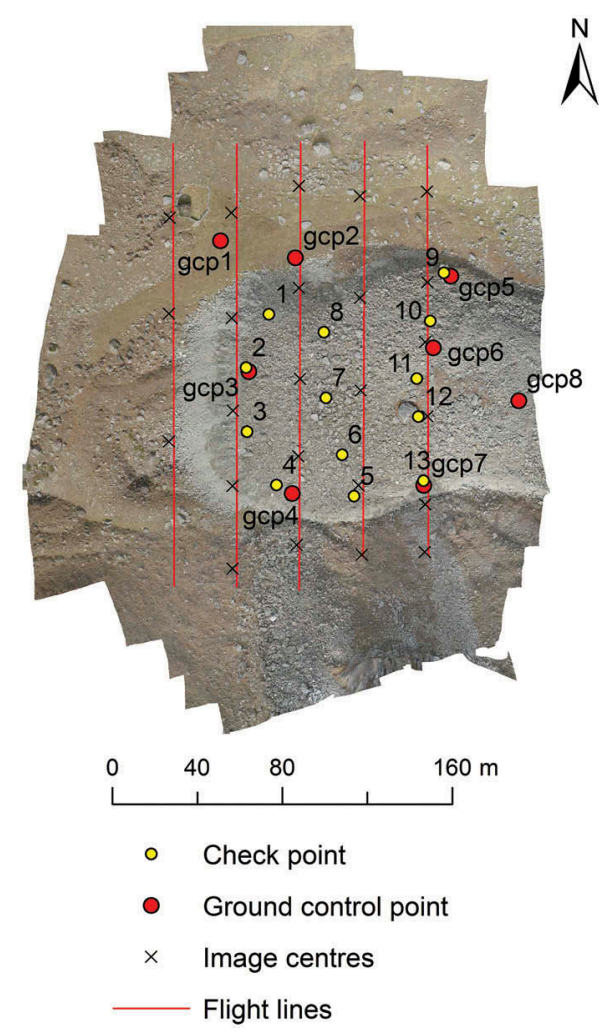

Figure 2. Distribution of the Ground Control Points (GCPs), the check points (CPs) and images along the flight lines, with the orthophoto mosaic as background.

circles with a drilled hole in the centre (Figure 3(b)). Differential post processing of the GNSS data was conducted using Trimble Business Center (TBC) v4 surveying software, linking the base station in the field with the permanent base station in Zermatt, $25 \mathrm{~km}$ away from the rock glacier. $x$ and $y$ values were referred to the Revised Swiss Reference System (CH1903+LV95) and elevation ( $z$ ) was recorded with respect to the Swiss Geoid Model Version 2004 (ChGeo2004). Mean horizontal and vertical precision were around 0.014 and $0.02 \mathrm{~m}$ for the GCPs and the CPs.

\subsection{Preparation and processing of the data}

Making the PhotoScan workflow (Figure 3) reproducible on ten model runs was done by indicating GCPs and CPs on images prior to importing them into PhotoScan. The same pixel was coloured with GNU Image Manipulation Program (GIMP). This way, GCPs and CPs could be indicated manually at the same location (1 pixel positioning accuracy) for each software run. First, the 24 images were imported in PhotoScan (Agisoft 2018), then the workflow in Figure 3 was followed. This workflow was repeated ten times, both in medium and high quality.

DSM accuracy was assessed through the RMSE of the CPs, that were introduced to verify whether the error in $z$ was in the same order of magnitude than that of the GCPs. This allows to detect systematic DSM deformations (James and Robson 2014). The variation between the 


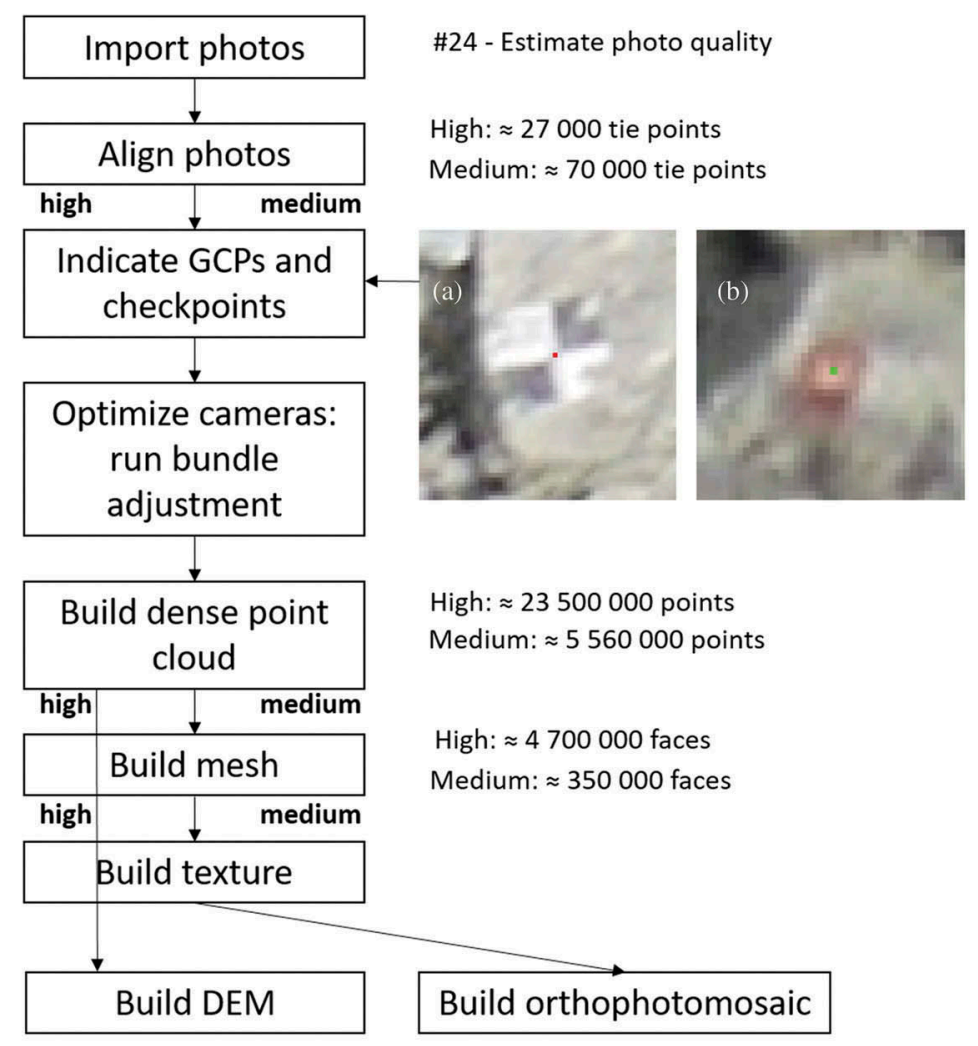

Figure 3. Workflow in PhotoScan (method based on Agisoft 2018). To enhance reproducibility, GCPs (a) and check points (b) were indicated on the images prior to the PhotoScan runs.

different model runs was assessed by calculating the standard deviation of both the $z$-value of each cell of the DSM and the calculated curvature (Zevenbergen and Thorne 1987), as a measure of the surface morphology, in ArcMap 10.3 (ESRI), using Cell Statistics (Spatial Analyst).

\section{Results}

The ten runs resulted in a $1.4 \mathrm{~cm}(=\mathrm{GSD})$ resolution DSM and a $3.14 \mathrm{~cm}$ orthophoto mosaic. The overall Root Mean Square Errors (RMSE) of $x, y$ and $z$ for the GCPs are of the same order of magnitude as for the CPs (Table 1). This suggests that the single model runs perform well. Moreover, the RMSE in Z for the CPs shows a vertical model accuracy of about $10 \mathrm{~cm}$ without DSM deformations. This single model accuracy is similar to accuracies reached in similar environments and surface roughness (Dall'Asta et al. 2017; Kenner et al. 2017).

The overall variation of the RMSE for the ten model runs, expressed as standard deviation (Table 1, Stdev), is considered small. The difference between the high-quality run and the medium-quality run is also small, especially in $z$, which is of most interest for volume calculations. This suggest that modelled coordinates differ little. Indeed, when the $x, y$, and $z$ coordinates of the different model runs are analysed, a standard deviation 
Table 1. Root Mean Square Errors (RMSE) of $x, y$ and $z$ to evaluate the overall model performance. The average and the standard deviation of the ten model runs are presented for both medium and high quality runs.

\begin{tabular}{llccccc}
\hline & & \multicolumn{2}{c}{ Medium } & & \multicolumn{2}{c}{ High } \\
\cline { 3 - 4 } \cline { 6 - 7 } & & Average $(\mathrm{cm})$ & Stdev $(\mathrm{cm})$ & & Average $(\mathrm{cm})$ & Stdev $(\mathrm{cm})$ \\
\hline GCPs & RMSE $(x)$ & 0.32 & 0.06 & & 6.78 & 0.07 \\
& RMSE $(y)$ & 2.50 & 0.01 & & 4.52 & 0.18 \\
\multirow{3}{*}{ CPs } & RMSE $(z)$ & 8.85 & 0.03 & & 8.56 & 0.26 \\
& RMSE $(x)$ & 11.84 & 0.07 & & 10.95 & 0.08 \\
& RMSE $(y)$ & 3.88 & 0.03 & & 5.05 & 0.07 \\
& RMSE $(z)$ & 9.96 & 0.20 & & 9.27 & 0.19 \\
\hline
\end{tabular}

below $5 \times 10^{-10} \mathrm{~m}$ for both high- and medium-quality runs was found. Variation between the coordinates of the different model runs is thus negligible.

To quantify the variation within the output products (DSMs z-value and curvature) of the ten model runs, the standard deviation for each cell value was calculated (Figure 4). Both the medium- and high-quality runs show more variation at the edges of the DSMs $(10 \mathrm{~cm}-3 \mathrm{~m})$ and curvature $(3000-4000$ within a range of $[-80000 ;+80000]$. This variation is due to less GCPs and less overlapping images (Figure 5), resulting in a lower point density and thus a higher model variation. The medium-quality run shows a clear pattern in variation with slope and surface roughness (Figure 4(a), (c); Figure 6), while the high-quality run has a more linear pattern of variation, which seems related to the flight lines and the overlap areas of the images, for both DSM and curvature (Figure 4(b), (d); Figure 6). Comparing the point clouds with the $\mathrm{M} 3 \mathrm{C} 2$ algorithm in CloudCompare (Lague, Brodu, and Leroux 2013) gives the same results, excluding the gridding process as cause of these model variations.

\section{Discussion and conclusion}

The variation between different PhotoScan model runs is relatively small, as shown by the very small difference between the calculated coordinates and small RMSE (Table 1). Nevertheless, different PhotoScan model runs can give different outcome products (in this case point clouds, DSMs and calculated curvatures), although same input data and processing details are used. The variation between DSMs is small and stays under the model accuracy for 88 to $96 \%$ of the study area (high- and medium-quality run respectively). However, in some cases the DSM variation is larger than the accuracy of the model itself $(\geq 10 \mathrm{~cm}$ in $z$ ), whereas 3 to $6 \%$ of these variations lie at the edge of the model (image overlap $<5$ ) and 1 to $6 \%$ lie within this boundary (high- and medium-quality run respectively).

DSM variation is clearly linked to slope gradient and surface roughness for the mediumquality run (Figure 4(c), Figure 6(c)). This is to be expected, since inaccuracies in $x$ and $y$ automatically resolve in inaccuracy in $z$, especially on steep slopes and areas with a high surface roughness. Curvature variation, as a measure on how well the model can reproduce the surface morphology, shows an even more pronounced relationship with slope gradient (Figure 4(a), Figure 6(a)). Standard deviations and thus variation in the model result are largest in areas with high slope gradient and surface roughness. This is especially relevant for geomorphic studies since process magnitude is higher on steep slopes, and model inaccuracies on these location may mislead interpretations. It is, therefore, recommended to add oblique viewpoint flights to get a higher point density and a better reproducibility by 


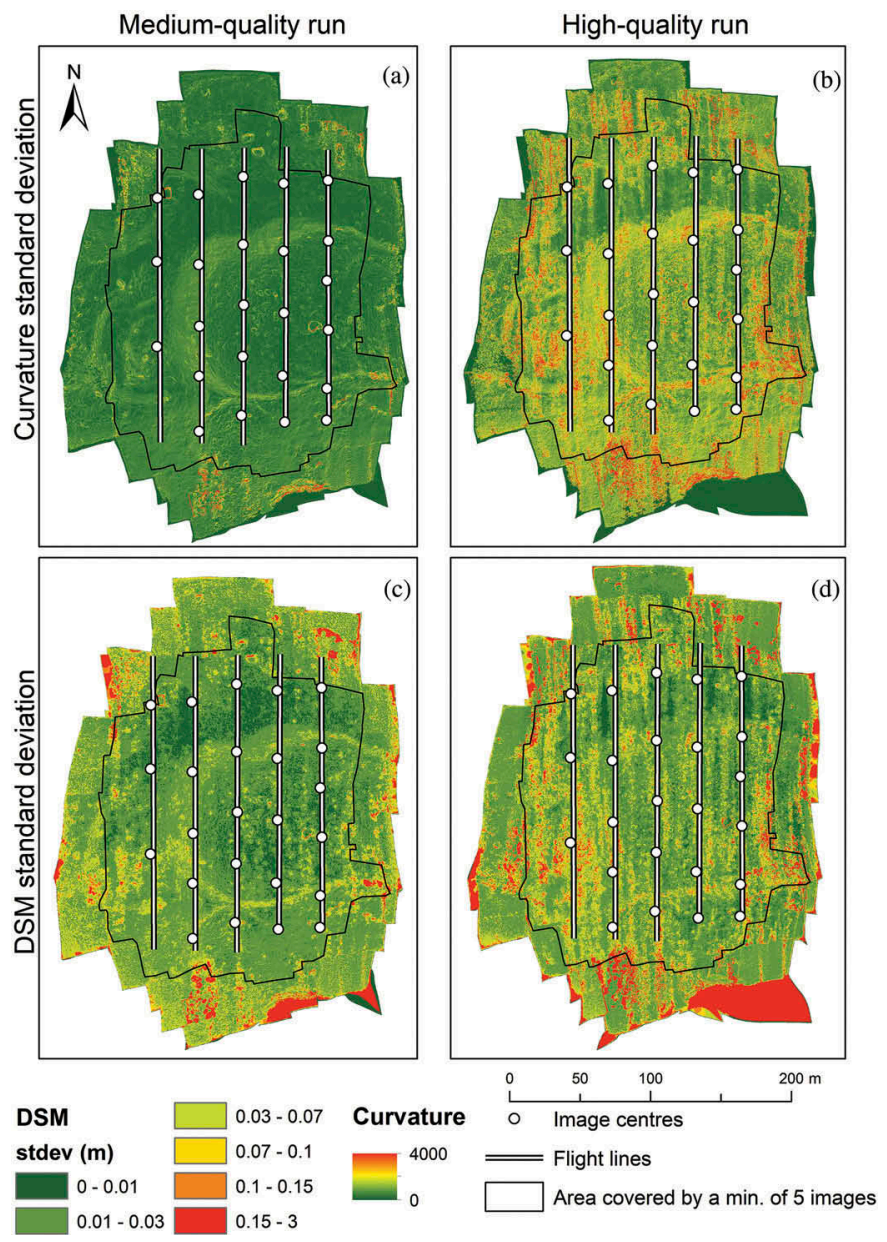

Figure 4. Standard deviations of the curvature value of each cell for the ten model runs in medium- (a) and high-quality run (b) and of the DSM value in $z$ of each cell for the ten model runs in medium- (c) and highquality run (d).

PhotoScan in such areas. Moreover, oblique viewpoint shots have been proven to avoid systematic DSM deformations caused by suboptimal camera calibration parameters (James and Robson 2014) and loss of accuracy due to sparse GCPs (Harwin, Lucieer, and Osborn 2015). A rapid increase in error with slope classes was also observed by Piermattei et al. (2016). This was due to areas with poor texture, like snow cover or shadow zones, which also result in a lower point density and can thus cause more model variation. However, rock glacier surface texture is primarily composed of different sizes of angular rocks (Ikeda and Matsuoka 2006) that can have low to medium albedo, well defined structures and medium to high contrast.

Step-like differences in DSMs were also found by James et al. (2017b), corresponding to changes in image overlaps. In order to find corresponding features, the software needs the feature to be visible on at least two images. Since image texture can differ greatly from case to case, it is difficult to advice a minimum number of overlapping images required for scene construction. Westoby et al. (2012) stated that a minimum of three overlapping images should be obtained. However, Figure 5 shows that variation in the modelled DSMs lowers significantly 


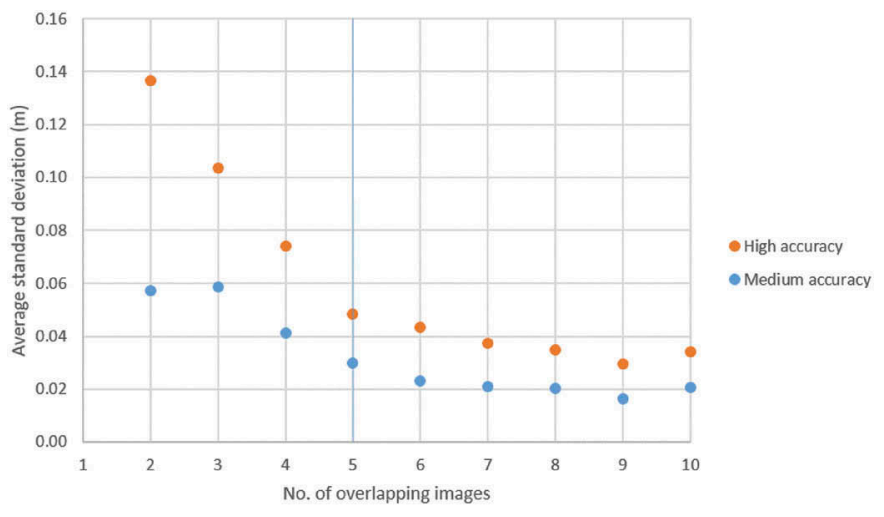

Figure 5. Variation of the model (standard deviation) as a function of the number of overlapping images.
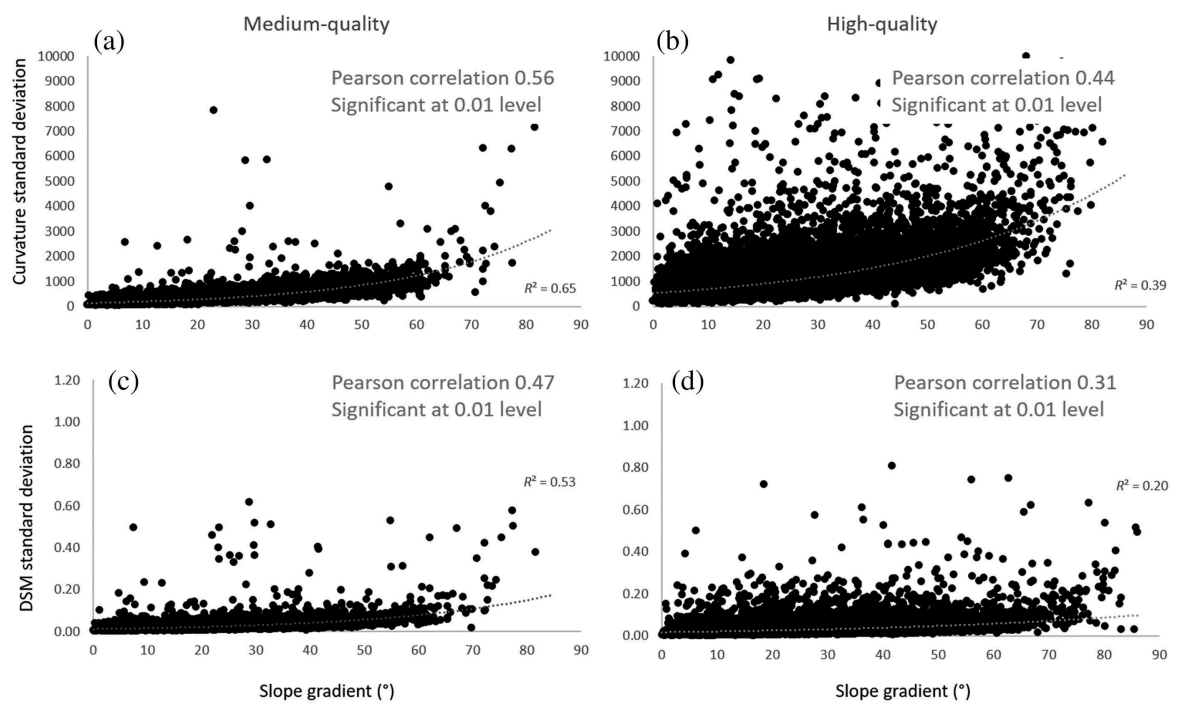

Figure 6. The exponential relationship between slope gradient and the variation in curvature $(a, b)$ and the variation in DSM (z) (c, d) for both medium- and high-quality runs, where the number of overlapping pictures is at least five. With $R^{2}$ as the coefficient of determination.

starting from five overlapping images. Flight planning is, therefore, very important and is usually done in multi-image blocks for an efficient aerial coverage (James and Robson 2014). Flight lines should exceed the area of interest to get optimal results.

A good distribution of GCPs is also advisable and part of the reason of a higher variability and a lower accuracy of the model at the edges. It will also minimize potential DSM deformations. Using CPs to validate the absolute model accuracy is also highly recommended (James and Robson 2014).

The overall model performance from the high- and medium-quality run lie very close to each other (Table 1, Figure 5). Especially the RMSE for $z$ is very similar. Considering the difference in computational effort calculation time (15 min vs. an hour), it is more efficient to use the medium-quality run in this case. Model variability is also more predictable for the medium- 
quality run, since it is more clearly linked to slope steepness. Moreover, both variations in DSM (z) and curvature show a better performance using the medium-quality run (Figure 6).

From the above we can conclude that PhotoScan is reliable software to perform high resolution processing of SFM-MVS data and that it can reproduce the same outcome with a minimum of variation, even in a complex topography. However, the model varies more at the model edges and in areas with pronounced/complex relief. Especially the latter may bias geomorphic interpretations. These variations can be minimized by using medium-quality runs, additional oblique viewpoints and realizing a minimum of five overlapping images. Therefore, good planning is crucial, especially when surveying large and complex terrain.

\section{Acknowledgments}

Adeline Frossard (Msc student UNIL) is thanked for her help during the field work. Field logistics were organized by IDYST-UNIL. The authors thank the anonymous reviewer for valuable comments.

\section{External links}

3D Model of this experiment: https://skfb.ly/6uM6w

Entire 3D model of the site: https://skfb.ly/6uNzZ

\section{Disclosure statement}

No potential conflict of interest was reported by the authors

\section{Funding}

H. Hendrickx's field stay was funded by Research Foundations - Flanders under a travel grant (V4.321.17N) for long research stay abroad.

\section{ORCID}

Sebastián Vivero (D) http://orcid.org/0000-0002-1813-9575

Philippe De Maeyer (D) http://orcid.org/0000-0001-8902-3855

\section{References}

Agisoft. 2018. "Agisoft PhotoScan User Manual." Professional Edition, Version 1.4. http://www. agisoft.com/downloads/user-manuals/

Dall'Asta, E., G. Forlani, R. Roncella, M. Santise, F. Diotri, and U. Morra Di Cella. 2017. "Unmanned Aerial Systems and DSM Matching for Rock Glacier Monitoring." ISPRS Journal of Photogrammetry and Remote Sensing 127: 102-114. doi:10.1016/j.isprsjprs.2016.10.003.

Delaloye, R., C. Lambiel, and G.-R. Isabelle. 2010. "Overview of Rock Glacier Kinematics Research in the Swiss Alps: Seasonal Rhythm, Interannual Variations and Trends over Several Decades." Geographica Helvetica 65 (2): 135-145. doi:10.5194/gh-65-135-2010.

Fonstad, M. A., J. T. Dietrich, B. C. Courville, J. L. Jensen, and P. E. Carbonneau. 2013. "Topographic Structure from Motion: A New Development in Photogrammetric Measurement." Earth Surface Processes and Landforms. doi:10.1002/esp.3366.

Frankl, A., C. Stal, A. Abraha, J. Nyssen, D. Rieke-Zapp, A. De Wulf, and J. Poesen. 2015. “Detailed Recording of Gully Morphology in 3D through Image-Based Modelling." CATENA 127 (April): 92-101. doi:10.1016/ j.catena.2014.12.016. 
Harwin, S., and A. Lucieer. 2012. "Assessing the Accuracy of Georeferenced Point Clouds Produced via Multi-View Stereopsis from Unmanned Aerial Vehicle (UAV) Imagery." Remote Sensing 4 (6): 15731599. doi:10.3390/rs4061573.

Harwin, S., A. Lucieer, and J. Osborn. 2015. "The Impact of the Calibration Method on the Accuracy of Point Clouds Derived Using Unmanned Aerial Vehicle Multi-View Stereopsis." Remote Sensing 7 (9): 11933-11953. doi:10.3390/rs70911933.

Ikeda, A., and N. Matsuoka. 2006. "Pebbly versus Bouldery Rock Glaciers: Morphology, Structure and Processes." Geomorphology 73 (3-4): 279-296. doi:10.1016/j.geomorph.2005.07.015.

James, M. R., and S. Robson. 2014. "Mitigating Systematic Error in Topographic Models Derived from UAV and Ground-Based Image Networks." Earth Surface Processes and Landforms 39 (10): 1413-1420. doi:10.1002/esp.3609.

James, M. R., S. Stuart Robson, S. D'Oleire-Oltmanns, and U. Niethammer. 2017a. “Optimising UAV Topographic Surveys Processed with Structure-from-Motion: Ground Control Quality, Quantity and Bundle Adjustment." Geomorphology 280: 51-66. doi:10.1016/j.geomorph.2016.11.021.

James, M. R., S. Robson, and M. W. Smith. 2017b. "3-D Uncertainty-Based Topographic Change Detection with Structure-from-Motion Photogrammetry: Precision Maps for Ground Control and Directly Georeferenced Surveys." Earth Surface Processes and Landforms 42 (12): 1769-1788. doi:10.1002/ esp.4125.

Javernick, L., J. Brasington, and B. Caruso. 2014. "Modeling the Topography of Shallow Braided Rivers Using Structure-from-Motion Photogrammetry." Geomorphology 213: 166-182. doi:10.1016/j. geomorph.2014.01.006.

Kenner, R., M. Phillips, C. Hauck, C. Hilbich, C. Mulsow, Y. Bühler, A. Stoffel, and M. Buchroithner. 2017. "New Insights on Permafrost Genesis and Conservation in Talus Slopes Based on Observations at Flüelapass, Eastern Switzerland." Geomorphology 290: 101-113. doi:10.1016/j.geomorph.2017.04.011.

Lague, D., N. Brodu, and J. Leroux. 2013. "Accurate 3D Comparison of Complex Topography with Terrestrial Laser Scanner: Application to the Rangitikei Canyon (N-Z)." ISPRS Journal of Photogrammetry and Remote Sensing 82: 10-26. International Society for Photogrammetry and Remote Sensing, Inc. (ISPRS). doi:10.1016/j.isprsjprs.2013.04.009.

Lannoeye, W., C. Stal, E. Guyassa, A. Zenebe, J. Nyssen, and A. Frankl. 2016. "The Use of SfMPhotogrammetry to Quantify and Understand Gully Degradation at the Temporal Scale of Rainfall Events: An Example from the Ethiopian Drylands." Physical Geography 37 (6): 430-451. doi:10.1080/02723646.2016.1234197.

Lowe, D. G. 2004. "Distinctive Image Features from Scale-Invariant Keypoints." International Journal of Computer Vision 60 (2): 91-110. doi:10.1023/B:VISI.0000029664.99615.94.

Niethammer, U., M. R. James, S. Rothmund, J. Travelletti, and M. Joswig. 2012. “UAV-Based Remote Sensing of the Super-Sauze Landslide: Evaluation and Results." Engineering Geology 128: 2-11. doi:10.1016/j.enggeo.2011.03.012.

Piermattei, L., L. Carturan, F. De Blasi, P. Tarolli, G. D. Fontana, A. Vettore, and N. Pfeifer. 2016. "Suitability of Ground-Based SfM-MVS for Monitoring Glacial and Periglacial Processes." Earth Surface Dynamics 4 (2): 425-443. doi:10.5194/esurf-4-425-2016.

Semyonov, D. 2011. "Algorithms Used in Photoscan [Msg 2]." www.agisoft.ru/forum/index.php? topic $=89.0$

Smith, M. W., J. L. Carrivick, and D. J. Quincey. 2015. "Structure from Motion Photogrammetry in Physical Geography." Progress in Physical Geography 40 (2): 247-275. doi:10.1177/0309133315615805.

Uysal, M., A. S. Toprak, and N. Polat. 2015. "DEM Generation with UAV Photogrammetry and Accuracy Analysis in Sahitler Hill." Measurement: Journal of the International Measurement Confederation 73: 539-543. doi:10.1016/j.measurement.2015.06.010.

Westoby, M. J., J. Brasington, N. F. Glasser, M. J. Hambrey, and J. M. Reynolds. 2012. "'StructureFrom-Motion' Photogrammetry: A Low-Cost, Effective Tool for Geoscience Applications." Geomorphology 179: 300-314. doi:10.1016/j.geomorph.2012.08.021.

Zevenbergen, L. W., and C. R. Thorne. 1987. "Quantitative Analysis of Land Surface Topography." Earth Surface Processes and Landforms 12 (1): 47-56. doi:10.1002/esp.3290120107. 